\title{
Biosynthesis of von Willebrand Protein by Human Megakaryocytes
}

\author{
Lee Ann Sporn, Stephen I. Chavin, Victor J. Marder, and Denisa D. Wagner \\ Hematology Unit, Department of Medicine, University of Rochester School of Medicine and Dentistry, Rochester, New York 14642
}

\begin{abstract}
Immunofluorescence staining of buffy coat smears from a patient with chronic myelogenous leukemia in accelerated phase showed that $\sim 13 \%$ of all nucleated cells contained von Willebrand protein and, therefore, appeared to be of megakaryocytic origin. This was confirmed by positive staining with antisera against platelet factor 4 and platelet glycoproteins. Short-term cultures of the buffy coat, which lacked endothelial cells, were metabolically labeled with [ ${ }^{35}$ S]methionine, and von Willebrand protein was immunopurified from cell lysates and culture medium. Cultures from this patient synthesized and secreted von Willebrand protein, in contrast with cultures from other patients with leukemia, who lacked circulating megakaryocytes, and from normal volunteers. The subunit composition of the megakaryocytic von Willebrand protein was very similar to that of human umbilical vein endothelial cells. The size of the processed subunit (220 $\mathrm{kD})$ and of the cellular $(260 \mathrm{kD})$ and secreted $(275 \mathrm{kD})$ precursors from the two cell types were indistinguishable by gel electrophoresis. Furthermore, the ratio of precursor to processed subunit and the pattern of cellular and secreted nonreduced multimers were very similar. It appears, therefore, that the processing steps in biosynthesis of von Willebrand protein used by the megakaryocytes are very similar to those of umbilical vein endothelial cells.
\end{abstract}

\section{Introduction}

von Willebrand $(\mathrm{vW})^{1}$ protein is a large, adhesive glycoprotein of complex multimeric structure that mediates the adhesion of platelets to subendothelium after injury (1). The absence of, or structural defects in $\mathrm{vW}$ protein result in the bleeding disorder $\mathrm{vW}$ disease caused by inadequate platelet binding at the injured site $(2,3)$. vW protein is synthesized by endothelial cells (4), which are likely to be the source of $\mathrm{vW}$ protein in plasma and subendothelium. In the endothelial cells, $\mathrm{vW}$ protein is stored in the Weibel-Palade bodies (5), a situation that allows for rapid release of the protein upon stimulation. $\mathrm{vW}$ protein is first synthesized as a $260-\mathrm{kD}$ precursor subunit that undergoes carbohydrate processing, disulfide bond formation to form large polymers, and proteolytic cleavage to the mature $220-\mathrm{kD}$ subunit form $(6,7)$. Small amounts of precursor with complex type carbohydrate are secreted $(275 \mathrm{kD})$ along with the processed (220

Address correspondence to Dr. Wagner, P.O. Box Med., University of Rochester Medical Center, 601 Elmwood Ave., Rochester, NY 14642.

Received for publication 26 December 1984 and in revised form 22 April 1985.

1. Abbreviations used in this paper: $\mathrm{CML}$, chronic myelogenous leukemia; $\mathrm{vW}$, von Willebrand.

J. Clin. Invest.

(c) The American Society for Clinical Investigation, Inc.

$0021-9738 / 85 / 09 / 1102 / 05 \quad \$ 1.00$

Volume 76, September 1985, 1102-1106
kD) subunit (6). While metabolically labeled, processed subunit has been purified from guinea pig megakaryocytes (8), the synthesis of precursor subunit, details of the processing steps, and information regarding multimer formation by these cells have not been described. An even greater lack of information exists for human megakaryocyte-derived $\mathrm{vW}$ protein. This is probably due to difficulties encountered in obtaining a sufficient number of megakaryocytes from human bone marrow to perform metabolic labeling studies. In this report, we show that human micromegakaryocytes present in the peripheral blood of a patient with chronic myelogenous leukemia (CML) in blast transformation synthesize, process, and secrete $\mathrm{vW}$ protein. The megakaryocyte-derived $\mathrm{vW}$ protein is very similar to that produced by human umbilical vein endothelial cells.

\section{Methods}

Studied patient. D.G. was a 39-yr-old white man in whom CML was diagnosed in 1975. At the time of diagnosis he had a white blood count

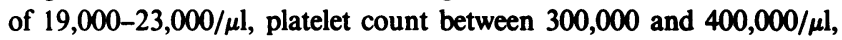
and leukocyte alkaline phosphatase of 10 . Peripheral blood leukocytes consisted of 50 segmented forms, 4 band neutrophils, 8 metamyelocytes, 3 myelocytes, 1 promyelocyte, 25 lymphocytes, 9 eosinophils, and 1 basophil. Philadelphia chromosome was present. His peripheral blood counts were controlled satisfactorily with oral Myleran, but in 1983 he developed symptoms relating to splenomegaly and was treated with 300 rad of splenic radiation. Leukopenia appeared after the radiation therapy, gradually disappearing as the spleen again enlarged. In January 1984, the patient underwent splenectomy for relief of abdominal pain and swelling. At this time the white blood count was $25,700 / \mu 1$, of which $38 \%$ were blasts. Platelet count was $440,000 / \mu 1$, but large degranulated forms were present as well as micromegakaryocytes. Bleeding time was $5 \frac{1}{2} \mathrm{~min}$. The blast cells were negative for terminal transferase and peroxidase, and the bone marrow revealed $50 \%$ blasts, increased megakaryocytes, and occasional micromegakaryocytes. Two blood samples were used for the studies reported here, obtained during this accelerated phase before treatment with hydroxyurea and low-dose subcutaneous cytosine arabinoside.

The leukemia was controlled only moderately well and the clinical course was characterized by progressively increasing bone pain and febrile episodes, requiring hospitalization in April 1984. At that time the white blood cell count was $57,700 / \mu$, with $10 \%$ segmented forms and $48 \%$ blasts, including many micromegakaryocytes. Platelet count was $182,000 /$ $\mu \mathrm{l}$ with some giant and bizarre forms. In addition, the smear also showed one nucleated red cell and hypersegmentation of the mature neutrophils. The patient's clinical condition continued to deteriorate during the next few months, and the white blood cell and platelet counts were treated empirically with hydroxyurea. At no time during his illness were there clinical episodes of thrombosis or bleeding. He died in June 1984.

Buffy coat and endothelial cell smears. Samples of 5-10 $\mathrm{ml}$ of heparinized blood were diluted $1 \mathrm{vol} / 3 \mathrm{vol}$ with McCoy's 5a medium (Flow Laboratories, Inc., McLean, VA), layered over one-third that total volume of Ficoll-Pacque (Pharmacia Fine Chemicals, Uppsala, Sweden), and centrifuged at $400 \mathrm{~g}$ for $30 \mathrm{~min}$. A small volume of the buffy coat preparation was removed and smeared onto $12 \times 12$-mm glass coverslips, air-dried for several minutes, then fixed in 3.7\% formaldehyde in phosphate-buffered saline (PBS) for $20 \mathrm{~min}$. Coverslips were rinsed once in PBS then stored at $4^{\circ} \mathrm{C}$ in PBS containing $1 \mathrm{mM}$ sodium azide. Endo- 
thelial cell smears were made in the same manner, using cells suspended by trypsinization from a flask of confluent endothelial cells obtained and cultured as previously described $(5,9)$.

Antisera. The preparation and characterization of antiserum used for immunofluorescence studies against human $\mathrm{vW}$ protein were as described previously (5). Antiserum against platelet factor 4 (10) and against platelet glycoproteins I, IIb, and IIIa (11) were kind gifts from Dr. Stephan Niewiarowski (Temple University Health Sciences Center, Philadelphia, PA) and Dr. Alan Gerwirtz (Temple University Health Sciences Center), respectively. Anti-vW protein antiserum used for immunoprecipitation was purchased from Calbiochem-Behring Corp. (La Jolla, CA).

Immunofluorescence. Fluorescence staining of buffy coat cells and of endothelial cells was carried out as previously described (5). Quantitation of the percentage of nucleated cells that stained positively with anti-vW protein antiserum was averaged from photographs of 12 random frames of stained buffy coat smears. Nucleated cells that stained as brightly as surrounding platelets were considered positive, and cells with lesser degrees of fluorescence were considered negative. The percentage of positive cells was calculated on the basis of the total number of nucleated cells in the frame.

Cell culture and labeling. Buffy coat cells were removed from FicollPaque by aspirating with a Pasteur pipette, counted, and suspended in $25-\mathrm{cm}^{2}$ tissue culture flasks (Nunc, Intermed, Denmark) at a concentration of $5 \times 10^{6}$ to $1 \times 10^{7}$ cells $/ \mathrm{ml}$ in 4-5 ml total volume of McCoy's 5 a medium containing $20 \%$ fetal bovine serum (Flow Laboratories, Inc.). Cultures were then placed in a $5 \% \mathrm{CO}_{2}$ high humidity incubator overnight, after which cells were removed and pelletized by centrifuging at $300 \mathrm{~g}$ for $5 \mathrm{~min}$. Cells were resuspended in medium containing $30 \mu \mathrm{Ci} / \mathrm{ml}$ ${ }^{35}$ S]methionine (Amersham Corp., Arlington Heights, IL) and again placed in a $5 \% \mathrm{CO}_{2}$ incubator for $36-48 \mathrm{~h}$.

Purification of $v W$ protein. At the end of the labeling period buffy coat cells were removed from flasks and pelletized by centrifugation as before. Media was removed and saved, and cells were washed once with PBS. Buffy coat cells were vortexed vigorously after addition of the lysis buffer (6). After lysing, the final concentration of ingredients was that of the radioimmunoprecipitation assay buffer used for washing the immunoprecipitate (12). The cell lysate and culture medium samples were incubated with gelatin Sepharose for $1.5 \mathrm{~h}$ at room temperature to remove fibronectin and other contaminating proteins that adhere nonspecifically. The gelatin Sepharose was removed by centrifugation. Protein A-Sepharose CL4B (30 mg) (Sigma Chemical Co., St. Louis, MO) was preincubated with $100 \mu \mathrm{l}$ anti-vW protein antiserum for $30 \mathrm{~min}$, then incubated with each sample for $1.5 \mathrm{~h}$ at room temperature. Protein A-Sepharose beads were washed extensively, then boiled in electrophoresis sample buffer (13) and analyzed by gel electrophoresis.

Electrophoresis gels. Sodium dodecyl sulfate (SDS)-polyacrylamide gels were prepared as described by Laemmli (13). Agarose horizontal slab gels were prepared using a solution of $2 \%$ agarose and $0.2 \%$ SDS in $0.05 \mathrm{M}$ phosphate buffer, $\mathrm{pH}$ 7.0. Densitometer scans of autoradiograms of agarose gels were made, and areas under peaks were measured by weighing cutout traces of the scans.

\section{Results}

Immunofluorescence staining of peripheral blood cells. Nucleated cells tentatively identified as micromegakaryocytes were seen in a Wright's stained preparation of a peripheral blood film obtained from the patient under study during the accelerated phase of his illness (Fig. 1). Immunofluorescence staining of the patient's buffy coat smears with anti-vW protein antiserum revealed positively stained nucleated cells (Fig. $2 a$ ). The granular pattern of staining was similar to that found in platelet alpha granules (Fig. $2, e$ and $f)$, which are known to contain $\mathrm{vW}$ protein $(14,15)$. The cells that exhibited positive staining were distinctly different from stained smears of endothelial cells (Fig. $2 d$ ) in that the peripheral blood cells were smaller, rounder, and had a higher nuclear/cytoplasmic ratio. Positive staining with anti-vW protein

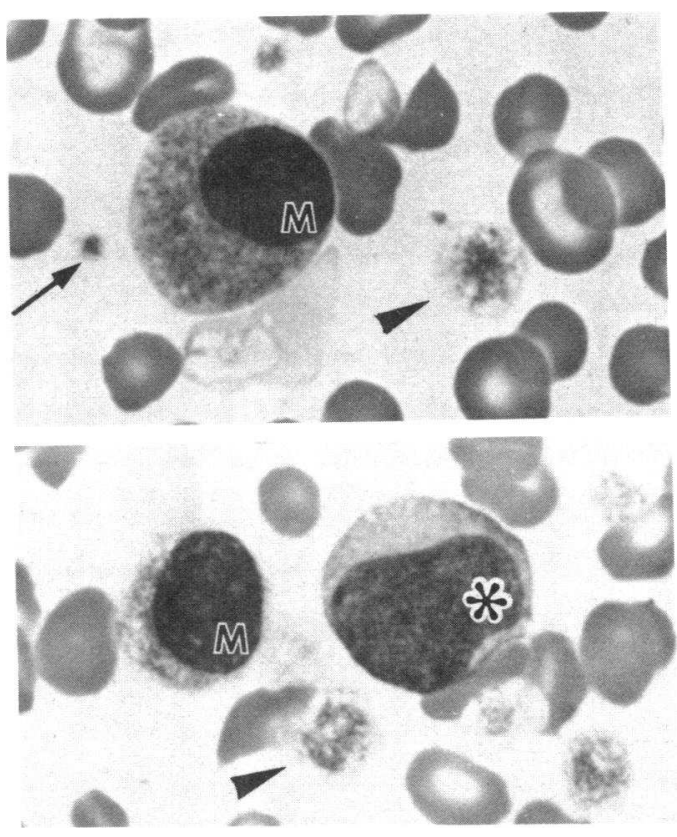

Figure 1. Wright's stained preparation of CML patient's peripheral blood smear. Giant platelets, some partially degranulated, as well as normal platelets are marked by arrowheads and small arrows, respectively. The cells with eccentrically located dense nuclei and granular cytoplasm that closely resembles that of platelets were tentatively identified by their appearance as micromegakaryocytes "M." A myeloblast is also present (asterisk).

antiserum was observed in nucleated cells with varying nuclear/ cytoplasmic ratio, including those with only a very small rim of cytoplasm (Fig. $2 f$ ). The percentage of nucleated cells that stained brightly with anti-vW protein antiserum was $13 \%$. No positively stained nucleated cells were seen in buffy coat smears from other patients with leukemia, two of whom had acute myelogenous leukemia and one of whom had acute lymphoblastic leukemia (not shown). No positive cells were seen when smears were stained with fluorescein isothiocyanate-conjugated antibody alone in the absence of anti-vW protein antiserum, indicating that positive staining was not due to $\mathrm{Fc}$ receptors present on the cell surface (Fig. $2 b$ ).

Staining of buffy coat smears with antiplatelet factor 4 antiserum also showed cells with a granular staining pattern that was similar to that obtained with anti-vW protein antiserum (Fig. 2 g). Bright, uniform (nongranular) staining was observed with antiserum against platelet glycoproteins (Fig. 2 h). Endothelial cells did not stain positively with either antiplatelet factor 4 or antiplatelet glycoprotein antisera (not shown). There were significantly fewer nucleated cells in relation to platelets in buffy coat smears from normal volunteers, and none of the nucleated cells stained positively with anti-vW protein antiserum (Fig. 2 c), antiplatelet factor 4 , or antiplatelet glycoprotein antiserum (not shown). These results further confirmed that nucleated cells of megakaryocytic origin were present only in the peripheral blood of the patient with CML.

$v W$ protein biosynthesis. Buffy coats were prepared from peripheral blood samples of the patient under study was well as from the other patients with leukemia and from normal volunteers. Cells were counted and suspended as described in Methods and placed in a $\mathrm{CO}_{2}$ incubator overnight before metabolic labeling with $\left[{ }^{35}\right.$ S $]$ methionine. Control cultures of $90 \%$ 

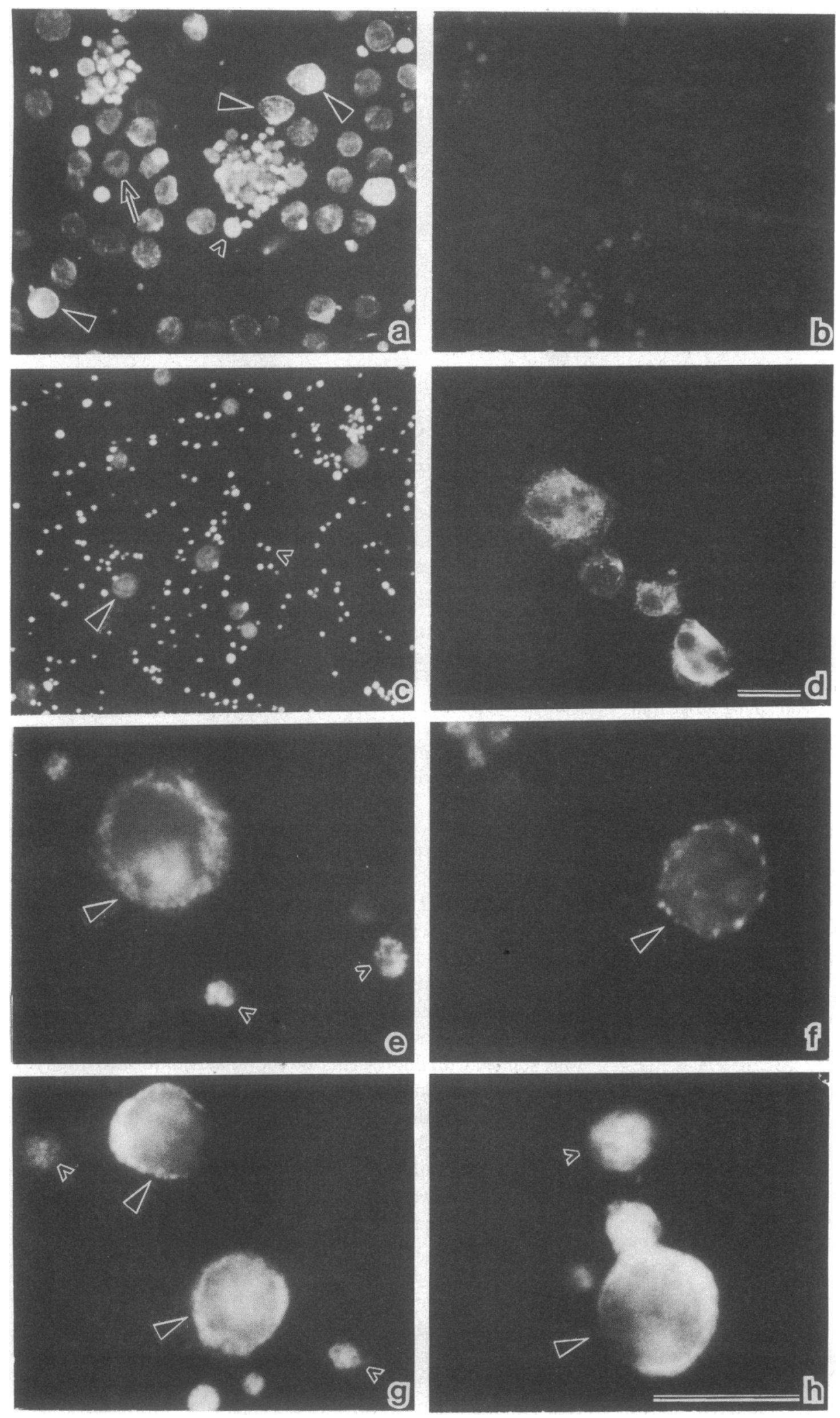

Figure 2. Immunofluorescent staining of buffy coat and endothelial cell smears. $a$ and $c-f$ were stained with anti-vW protein antiserum. $a$ and $b$ represent buffy coat smears from the CML patient. (a) Large arrowheads point to some of the nucleated cells that were judged as positively stained. The small arrowhead points to an abnormal giant platelet. The arrow indicates an example of an unstained cell. (b) Control staining with fluorescein isothiocyanate-conjugated antibody in the absence of first antisera, demonstrating that the positive staining in $(a)$ is not due to the presence of Fc receptors on these cells. (c) Buffy coat smear from a healthy volunteer showing positive staining only of platelets (small arrowhead). Large arrowhead points to an unstained, nucleated cell. (d) Smear of normal human umbilical vein endothelial cells printed at the same magnifcation as $a-c$, showing that these cells are larger and less uniform in shape than positively stained nucleated cells in $a . e-h$ are higher magnification photographs of cells from the CML patient. $e$ and $f$ show the presence of $\mathrm{vW}$ protein in granules of cells with very different nuclear/cytoplasmic ratios (large arrowheads). Small arrowheads point to platelets with a similar granular-staining pattern. $(g)$ Staining with antiplatelet factor 4 antiserum reveals a granular-staining pattern similar to that observed with anti-vW protein antiserum in nucleated cells (large arrowheads) and in platelets (small arrowheads). ( $h$ ) Treatment with antiserum prepared against platelet surface glycoproteins produced a uniform staining pattern in nucleated cells (large arrowheads) and in platelets (small arrowheads). Bar in $a-d, 20 \mu \mathrm{m}$; bar in $e-$ $h, 10 \mu \mathrm{m}$. confluent human umbilical vein endothelial cells were labeled in parallel. During the culture time of the buffy coat cells no substantial cell division was observed. Cells settled to the bottom of the flask, and platelet clumps formed (Fig. $3 a$ ), but no adherent or spread cells that resembled endothelial cells (Fig. $3 b$ ) were observed even after $6 \mathrm{~d}$ in culture. At the end of the 3648-h labeling period, all culture media were collected and cells were lysed.
vW protein was immunopurified from the culture medium and cell lysates and analyzed on gels. In the buffy coat cultures, radiolabeled $\mathrm{vW}$ protein was detected only in the samples obtained from the CML patient, indicating that it was synthesized by the megakaryocytes. The $\mathrm{vW}$ protein subunit composition and migration on reduced polyacrylamide gels were indistinguishable from endothelial cell $\mathrm{vW}$ protein composition and migration (Fig. 4). Both cell types synthesized the finished 220- 

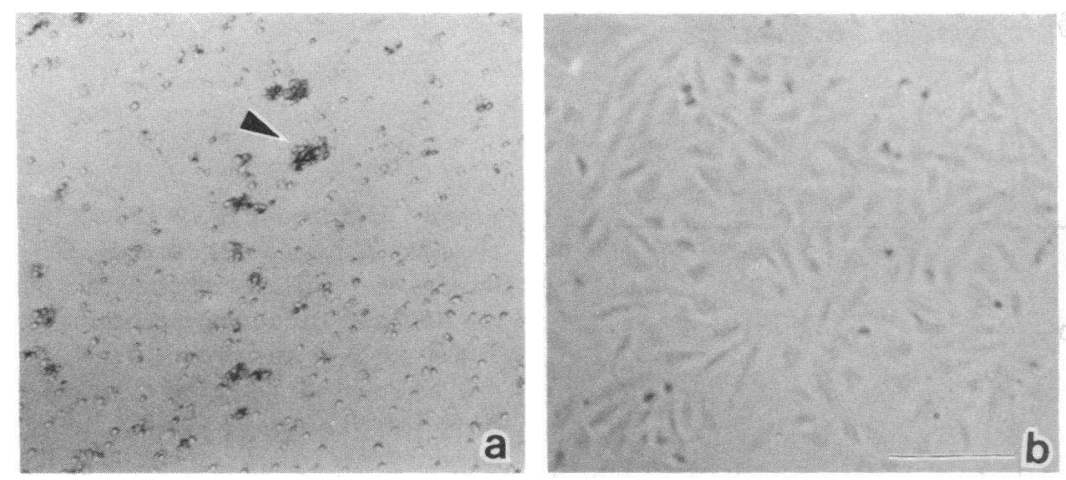

Figure 3. Cultures of buffy coat and endothelial cells. (a) Buffy coat cells from CML patient after $6 \mathrm{~d}$ in culture. No adherent and spread cells are seen. Arrowhead points to a platelet clump. (b) Normal human umbilical vein endothelial cells. Unlike buffy coat cells, endothelial cells are not round and are well attached to the tissue culture flask. Bar is $100 \mu \mathrm{m}$.

$\mathrm{kD}$ subunit and the $260-\mathrm{kD}$ intracellular precursor, and both secreted small amounts of the slightly larger $(275 \mathrm{kD})$ precursor form.

Analysis of the nonreduced $\mathrm{vW}$ protein on agarose gels showed that the dimer and higher molecular weight multimers of $\mathrm{vW}$ protein from megakaryocytes migrated to the same positions in the gel as those from endothelial cells (Fig. 5). However, we have observed slightly less dimer in comparison to the amount of higher molecular weight multimers in the megakaryocytes and in their secreted $\mathrm{vW}$ protein than was present within endothelial cells and in endothelial cell culture medium (Figs. 5 and 6).

\section{Discussion}

Immunofluorescence microscopy revealed the presence of $\mathrm{vW}$ protein in small and large nucleated cells in buffy coat smears from a patient with accelerated phase CML (Fig. $2 a, e$, and $f$ ), suggesting that the cells were of megakaryocytic origin. This was supported by positive staining of these cells with antisera against platelet factor 4 and against platelet glycoproteins (Fig. $2 g$ and $h$ ). The apearance of these megakaryocytic cells is not an uncommon occurrence in acute leukemia. A recent study showed that 12 patients with acute myelogenous leukemia, which represented $11.8 \%$ of the group studied, had circulating megakar-

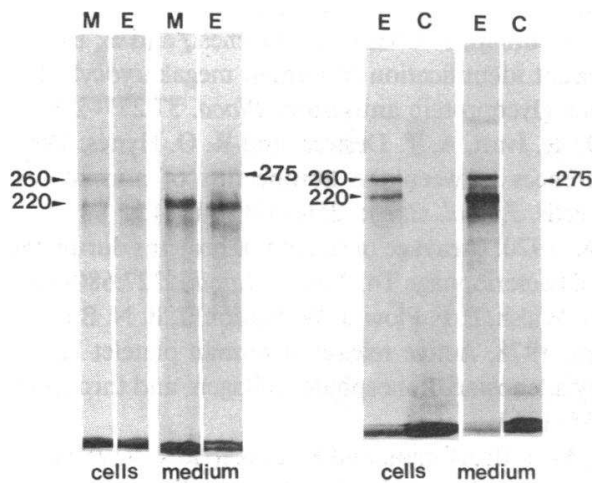

Figure 4. Subunit composition of $\mathrm{vW}$ protein from megakaryocyte (M) and endothelial cell (E) cell lysates and culture media. Cells were metabolically labeled with $\left[{ }^{35} \mathrm{~S}\right]$ methionine, and immunopurified $\mathrm{vW}$ protein was analyzed, reduced on $5 \%$ polyacrylamide gels. The $260-$ $\mathrm{kD}$ precursor and $220-\mathrm{kD}$ processed subunits were present in both megakaryocyte and endothelial cell lysates (cells). The slightly larger 275-kD precursor subunit along with the processed 220-kD subunit were also secreted by both cultures (medium). No detectable vW protein was synthesized by control buffy coat cultures from normal volunteers (C). yocytes as a result of transformation of the megakaryocytic progenitor line (16). About $13 \%$ of nucleated cells of varying size in our patient's blood stained positively with anti-vW protein antiserum. $\mathrm{vW}$ protein-containing granules were observed even in megakaryocytes with a very high nuclear/cytoplasmic ratio.

Metabolic labeling of buffy coat cultures demonstrated that vW protein was synthesized by the patient's circulating cells. Endothelial cells were not found in the buffy coat of the studied patient, nor in the buffy coat of normal controls nor in other patients with leukemia. Furthermore, even after several days of culture, no attached or spread cells were seen (Fig. $3 a$ ), making it unlikely that $\mathrm{vW}$ protein biosynthesis was due to the presence of endothelial cells. Buffy coats from normals and from the other leukemia patients contained no nucleated cells that stained positively with anti-vW protein antiserum, and their cultures revealed no biosynthesis of $\mathrm{vW}$ protein. Although the megakaryocyte-derived $\mathrm{vW}$ protein resembled that of endothelial cells, it contained relatively less dimer, supporting our conclusion that the $\mathrm{vW}$ protein synthesized by the CML patient buffy coat culture was not of endothelial origin. It is unlikely that platelets synthesized the $\mathrm{vW}$ protein since they possess only vestigial protein synthetic capacity and since control cultures containing platelets showed no such synthesis. Therefore, the synthesis of $\mathrm{vW}$ protein by the buffy coat cultures from the patient studied was due to the presence of micromegakaryocytes.

The processing steps used by the megakaryocytes in the biosynthesis of $\mathrm{vW}$ protein are likely to be similar to those described for endothelial cells $(7,17)$. Like endothelial cells, megakaryocytes contain an intracellular pool of a $260-\mathrm{kD}$ precursor subunit and a pool of dimer molecules. As for endothelial cells, some precursor of larger molecular size $(275 \mathrm{kD})$ was secreted, suggesting that the difference between megakaryocyte intracellular and secreted precursor could also have been due to carbohydrate processing, as has been reported for endothelial cell precursor

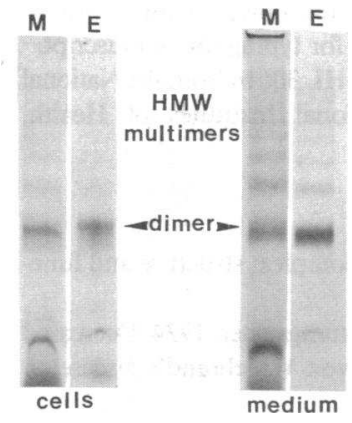

Figure 5. Comparison of $\mathrm{vW}$ protein polymers synthesized by megakaryocyte $(M)$ and by endothelial cells (E). Purified, metabolically labeled vW protein was analyzed, nonreduced, on $2 \%$ agarose gels. Although the dimer is the major species synthesized by the endothelial cells and the megakaryocytes, higher molecular weight (HMW) multimers are also produced by both cultures, and they co-migrate on these gels. (Figures are composed of different autoradiographic exposures of the same gel.) 

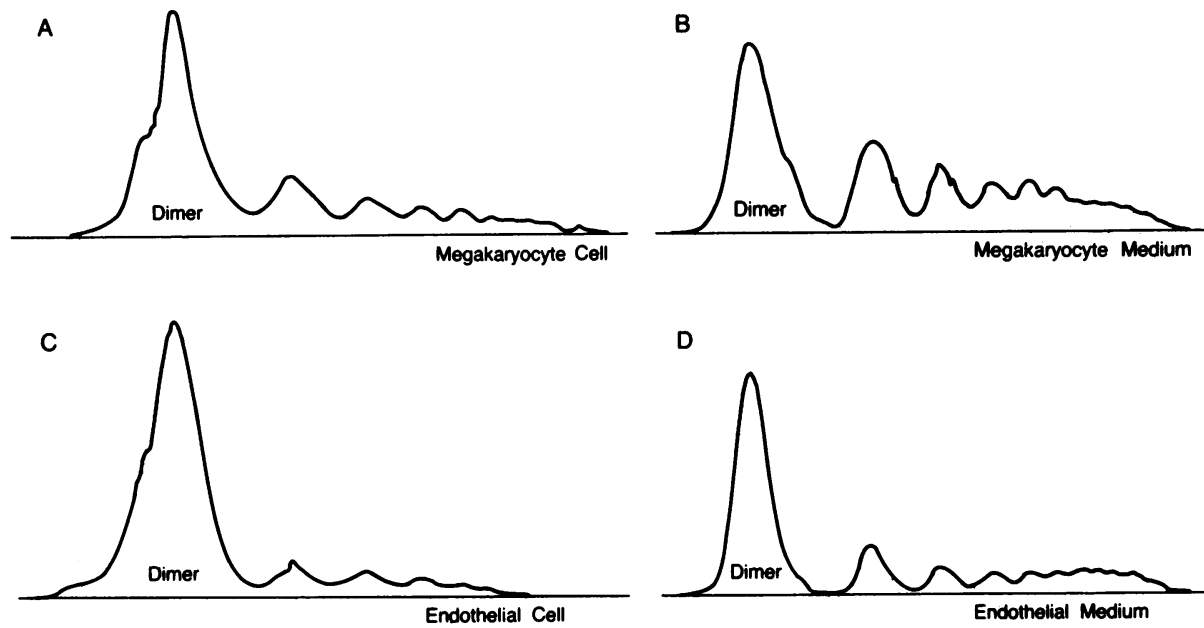

D

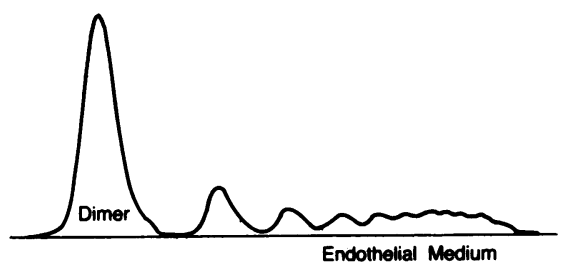

Figure 6. Distribution of $\mathrm{vW}$ protein between the different polymeric sizes. The tracings represent densitometric scans of gels from a representative experiment. The dimer band represents $61 \%$ of the total area above the base line in megakaryocyte cell lysate $(A)$, and $45 \%$ in megakaryocyte cell culture medium $(B)$, whereas the dimer composes $81 \%$ and $58 \%$ of the total area in endothelial cell lysates $(C)$ and endothelial cell culture medium $(D)$, respectively.
(17). The presence of multimers in the megakaryocyte cell lysate suggests that the polymerization of $\mathrm{vW}$ protein was intracellular, as is the case for endothelial cells (6). The same migration pattern of the polymers on nonreduced gels (Fig. 5) suggests that the interchain and intrachain disulfide bonds are similar to those of endothelial cell $\mathrm{vW}$ protein. The only possible difference between megakaryocyte and endothelial cell $\mathrm{vW}$ protein biosynthesis detected in this study was in the extent of interdimer disulfide bond formation. This difference was observed in three different experiments, but from only one study could we obtain complete, quantitative results (Fig. 6). The percentage of endothelial $\mathrm{vW}$ protein present as dimer (Fig. 6) agrees closely with results reported by Lynch et al. (18) determined from quantitation of radioactivity in gel slices. The reason that the interdimer disulfide bond formation processing step, which is localized to the Golgi and post-Golgi compartments in the endothelial cell (17), appears to be carried out more extensively in the megakaryocytes is not known, nor do we know if this difference is related to the leukemic origin of these cells.

To summarize, we have demonstrated $\mathrm{vW}$ protein biosynthesis by megakaryocytic cells in the peripheral blood of a patient with CML in accelerated phase. Fluorescence staining and metabolic labeling techniques showed that the megakaryocytes synthesized, processed, stored, and secreted $\mathrm{vW}$ protein that contained the same precursors and cleaved subunits as $\mathrm{vW}$ protein produced by human endothelial cells. The building block of $\mathrm{vW}$ protein polymers appears to be a dimer in the megakaryocyte, and its polymerization, though possibly yielding a slightly larger proportion of high molecular weight multimers than found in the endothelial cells, is likely to be intracellular.

\section{Acknowledgments}

We would like to thank Dr. John P. Olson for the opportunity to study this patient, Dr. Stephan Niewiarowski and Dr. Alan Gewirtz for generously providing us with antisera, Dr. Joanne Janas for the photomicrographs, and Carol Weed and Beth Bush for typing the manuscript.

This work was supported in part by grant HL 30616 from the National Heart, Lung, and Blood Institute, National Institutes of Health, Bethesda, MD.

\section{References}

1. Hoyer, L. W. 1981. The Factor VIII complex: structure and function. Blood. 58:1-13.

2. Tschop, T. B., H. J. Weiss, and H. R. Baumgartner. 1974. Decreased adhesion of platelet to subendothelium in von Willebrand's disease. $J$. Lab. Clin. Med. 83:296-300.
3. Sakariassen, K. S., P. A. Bolhuis, and J. J. Sixma. 1979. Human blood platelet adhesion to artery subendothelium is mediated by Factor VIII-von Willebrand factor bound to the subendothelium. Nature (Lond.). 279:636-638.

4. Jaffe, E. A., L. W. Hoyer, and R. L. Nachman. 1973. Synthesis of antihemophilic factor antigen by cultured human endothelial cells. $J$. Clin. Invest. 52:2757-2764.

5. Wagner, D. D., J. B. Olmsted, and V. J. Marder. 1982. Immunolocalization of von Willebrand protein in Weibel-Palade bodies of human endothelial cells. J. Cell Biol. 95:355-360.

6. Wagner, D. D., and V. J. Marder. 1983. Biosynthesis of von Willebrand protein by human endothelial cells: identification of a large precursor polypeptide chain. J. Biol. Chem. 258:2065-2067.

7. Lynch, D. C., R. Williams, T. S. Zimmerman, E. P. Kirby, and D. M. Livingston. 1983. Biosynthesis of the subunit of Factor VIIIR by bovine aorta endothelial cells. Proc. Natl. Acad. Sci. USA. 80:27382742.

8. Nachman, R., R. Levine, and E. A. Jaffe. 1977. Synthesis of Factor VIII antigen by cultured guinea pig megakaryocytes. J. Clin. Invest. 60: 914-921.

9. Gimbrone, M. A., Jr., R. S. Cotron, and J. Folkman. 1974. Human vascular endothelial cells in culture. Growth and DNA synthesis. J. Cell Biol. 60:673-684.

10. Rucinski, B., S. Niewiarowski, P. James, D. A. Walz, and A. Z. Budzynski. 1979. Antiheparin proteins secreted by human platelets. Purification, characterization, and radioimmunoassay. Blood. 53:47-51.

11. Mazur, E., R. Hoffman, J. Chasis, S. Marchesi, and E. Bruno. 1981. Immunofluorescent identification of human megakaryocyte colonies using anti-platelet glycoprotein antiserum. Blood. 57:277-280.

12. Wagner, D. D., R. Ivatt, A. T. Destree, and R. O. Hynes. 1981. Similarities and differences between the fibronectins of normal and transformed hamster cells. J. Biol. Chem. 256:11708-11715.

13. Laemmli, U. K. 1970. Cleavage of structural proteins during the assembly of the head of bacteriophage T4. Nature (Lond.). 227:680-685.

14. Koutts, J., P. N. Walsh, E. F. Plow, J. W. Fenton II, B. N. Bouma, and T. S. Zimmerman. 1978. Active release of human platelet Factor VIII-related antigen by adenosine diphosphate, collagen, and thrombin. J. Clin. Invest. 62:1255-1263.

15. Zucker, M. B., M. J. Broekman, and K. L. Kaplan. 1979. Factor VIII related antigen in human blood platelets. Localization and release by thrombin or collagen. J. Lab. Clin. Med. 94:675-682.

16. Huang, M., C. Li, W. L. Nichols, J. Young, and J. A. Katzmann. 1984. Acute leukemia with megakaryocytic differentiation: a study of 12 cases identified immunocytochemically. Blood. 64:427-439.

17. Wagner, D. D., and V. J. Marder. 1984. Biosynthesis of von Willebrand protein by human endothelial cells: processing steps and their intracellular localization. J. Cell Biol. 99:2123-2130.

18. Lynch, D. C., T. S. Zimmerman, E. P. Kirby, and D. M. Livingston. 1983. Subunit composition of oligomeric human von Willebrand factor. J. Biol. Chem. 258:12757-12760. 